\section{REVISTA}

Actualidades Investigativas en Educación

\title{
ANTECEDENTES INTERNACIONALES Y NACIONALES DE LAS TIC A NIVEL SUPERIOR: SU TRAYECTORIA EN PANAMÁ
}

INTERNATIONAL AND NATIONAL POLICIES OF THE ICTS IN HIGHER EDUCATION: ITS IMPLICATIONS IN PANAMA

\section{Volumen 12, Número 3 \\ Setiembre-Diciembre}

pp. $1-25$

Este número se publicó el 30 de setiembre de 2012

Olda María Cano Lassonde

Revista indizada en REDALYC

Revista distribuida en las bases de datos:

CATÁLOGO DE LATINDEX, IRESIE, CLASE, DIALNET, DOAJ, E-REVIST@S,

Revista registrada en los directorios:

ULRICH'S, REDIE, RINACE, OEI, MAESTROTECA, PREAL, CLASCO 


\title{
ANTECEDENTES INTERNACIONALES Y NACIONALES DE LAS TIC A NIVEL SUPERIOR: SU TRAYECTORIA EN PANAMÁ \\ INTERNATIONAL AND NATIONAL POLICIES OF THE ICTS IN HIGHER EDUCATION: ITS IMPLICATIONS IN PANAMA
}

\author{
Olda María Cano Lassonde ${ }^{1}$ \\ "El docente de hoy debe permanecer en una constante actualización de conocimientos \\ y competencias durante toda su carrera, debido a que la educación pareciera estar en \\ proceso de una transformación radical, como consecuencia de la irrupción de las TIC \\ en el ámbito educativo". \\ Giugni y Araujo, 2010
}

Resumen: El presente documento recoge los aportes de una investigación titulada: "Modelo de Aprendizaje en Entornos Virtuales en la Educación Superior" que se está desarrollando para el Doctorado en Investigación con énfasis en las Ciencias Sociales en la Universidad Autónoma de Chiriquí (UNACHI), David, Chiriquí, República de Panamá, cuyo Programa Doctoral se realiza de acuerdo con el convenio firmado entre UNACHI y la Universidad del Mar, Chile. Se destacan las gestiones más sobresalientes y las acciones dirigidas hacia la implementación de las TIC para mejorar la calidad de la educación a nivel superior. Se describen los cambios generados por las nuevas Tecnologías de la Información y de la Comunicación en el sistema educativo a nivel mundial, vaticinando la transformación del proceso de enseñanza-aprendizaje, y la forma en que docentes y alumnos acceden al conocimiento y la información. Finalmente, se puntualiza el esfuerzo que realiza el Estado Panameño en la introducción de las TIC a nivel superior con una descripción de la trayectoria de la educación virtual en diferentes instituciones a nivel nacional.

Palabras clave: TECNOLOGÍAS DE LA INFORMACIÓN Y COMUNICACIÓN (TIC), EDUCACIÓN A NIVEL SUPERIOR, PROCESO ENSEÑNANZA-APRENDIZAJE, CALIDAD DE LA EDUCACIÓN, POLÍTICAS INTERNACIONALES Y NACIONALES, EDUCACIÓN VIRTUAL, PANAMÁ

\begin{abstract}
The following paper reflects input of a research study entitled "A Model for Virtual Learning in Higher Education", which is being developed for a Doctoral Program in Research with emphasis in Social Science at Universidad Autónoma de Chiriquí (UNACHI), David, Chiriquí, Republic of Panama. This doctoral program is being held according to the agreement signed between UNACHI and Universidad del Mar, Chile. This article makes reference to the international and national policies, particularly in the Republic of Panama, for the implementation of the Information and Communication Technologies (ICTS). The paper highlights the most outstanding management policies and directed actions towards the execution of ICTs for the improvement of the quality of higher education. The main changes or transformations generated from ICTs are described, foreseeing renovations in the teaching and learning process and in the ways both professors and students acquire knowledge and information in higher education. Finally, the paper pinpoints the efforts done by the Panamanian government to innovate in virtual education through ICTs nationwide.
\end{abstract}

Key words: INFORMATION AND COMMUNICATION TECHNOLOGIES (ICTS), HIGHER EDUCATION, TEACHING AND LEARNING PROCESS, QUALITY OF EDUCATION, INTERNATIONAL AND NATIONAL POLICIES, VIRTUAL EDUCATION, PANAMA

\footnotetext{
1 Profesora Regular Titular, Tiempo Completo, de la Universidad Autónoma de Chiriquí (UNACHI), Panamá. Doctoranda- Doctorado en InvestigaciónUNACHI-Universidad del Mar de Chile; Maestría en Inglés con énfasis en Metodología de la Enseñanza; Maestría y Postgrado en Docencia Superior, Licenciada en la Enseñanza del Inglés; Vice-Decana, Facultad de Humanidades, UNACHI.
}

Dirección electrónica: oldamaria.cano@gmail.com

Ensayo recibido: 10 de abril, 2012

Aprobado: 20 de setiembre, 2012 


\section{Introducción}

El mundo globalizado de hoy ha generado transformaciones en la educación. Estas transformaciones han permitido la incorporación de las Tecnologías de la Información y de la Comunicación (TIC) para el establecimiento de universidades virtuales con la creación de cursos y programas a distancia. Sin embargo, la virtualización de la educación ha dado paso a una serie de críticas sobre su eficiencia y eficacia. Las críticas giran alrededor de las experiencias de aprendizajes virtuales y cuestionan su valor en la generación de aprendizajes significativos y permanentes. Otro aspecto controversial hace referencia a la calidad académica, ya que involucra un cambio de paradigmas pedagógicos y didácticos en la presentación y adquisición de la información, en las competencias requeridas tanto para el docente y para el estudiante, en el cambio de roles, entre otros.

Este escepticismo se origina a raíz del desafío que enfrentan los sistemas educativos de todo el mundo para la utilización de las Tecnologías de la Información y de la Comunicación (TIC) de manera que estas funcionen como un medio o recurso para brindarle al estudiantado las herramientas y conocimientos necesarios que se requieren en el siglo $X X I$, que les permita ser exitosos y competitivos en su vida personal y profesional, y que les abra las puertas en su integración al mundo globalizado donde se relacionan y conviven. Estas herramientas y recursos incluyen tanto la alfabetización inter y transdisciplinaria como la alfabetización digital.

A pesar del recelo hacia la tecnoeducación, el uso pedagógico de las TIC en la educación superior está cambiando radicalmente la manera en que los docentes enseñan, por un lado. Por otro, las TIC han tenido sus efectos en el aprendizaje de los alumnos tanto en el modo en que estudian, aprenden, investigan, trabajan, se comunican e interactúan entre sí, como en las estrategias que utilizan para la elaboración, producción, y construcción y adquisición de sus propios conocimientos. Siendo esta comunicación la primera parte de la tesis doctoral titulada: "Modelo de Aprendizaje en Entornos Virtuales a Nivel Superior", tanto las críticas como las controversias sobre el aprendizaje significativo generados en la utilización de los entornos virtuales se abordarán y aclararán en la segunda publicación, durante el progreso de esta tesis doctoral, que se enfoca en el fundamento epistemológico y pedagógico de los entornos virtuales a nivel superior. Por lo tanto, es primordial abordar los antecedentes internacionales y nacionales de las TIC para tener un mejor entendimiento 
sobre su aplicación en los últimos años, dada su conexión directa con los entornos virtuales de aprendizaje.

En efecto, las TIC conducen a nuevos modelos educativos a nivel universitario y plantean la necesidad de pasar de modelos tradicionales de enseñanza a modelos innovadores de aprendizaje centrados en el alumno a través del aprendizaje virtual. Con la llegada de las tecnologías, el énfasis de la profesión docente se transforma de un enfoque centrado en el profesor que se basa en prácticas magistrales, o el discurso, hacia una formación centrada, en particular, en el alumno dentro de un entorno interactivo de aprendizaje. Esta interactividad, que promueven las TIC, se encamina a mejorar la calidad de la educación por medio de la diversidad de contenidos. Los modelos centrados en el alumno, al que se hace mención, promueven el aprendizaje colaborativo en línea, la experimentación e investigación, la formación de comunidades virtuales como comunidades de intercambio, el incremento de la autonomía y la responsabilidad del alumno respecto al propio proceso de aprendizaje.

Como consecuencia, la construcción de conocimientos se realiza en forma colectiva, participativa, dinámica, activa, colaborativa y critico-reflexiva. Con los modelos centrados en el alumno, conducidos por las TIC, se demanda más del estudiantado, y estos ponen en práctica las habilidades de pensamiento de orden superior o la meta cognición, como el pensamiento crítico, el pensamiento sistémico, análisis, síntesis, evaluación, entre otros. Quesada (2010) ha indicado, muy puntualmente, que la implementación de los entornos virtuales de aprendizaje mediados por las TIC contribuyen a una verdadera alfabetización científica y tecnológica, tanto de estudiantes como de docentes, con un logro más eficiente del conocimiento, con un desarrollo de competencias comunicativas e investigativas y con mayores retos de las potencialidades del alumno hacia un aprendizaje autónomo y colaborativo. Esta nueva modalidad abre un mundo de posibilidades a nivel superior: al aprendizaje mixto (b-learning), el aprendizaje virtual (elearning) y la enseñanza flexible.

Sin lugar a dudas, las TIC tienen, hoy por hoy, muchas aplicaciones y usos, debido a la gran cantidad de información que se ha generado, y a la tecnología para disponer de ella, por eso, los profesores, más que depositarios y remisores del conocimiento, deben ser facilitadores para adquirir aprendizajes, y para facilitar a los estudiantes nuevos conocimientos. 
En síntesis, en esta comunicación se describen las políticas internacionales y las nacionales, particularmente en la República de Panamá, sobre la importancia e implementación de las Tecnologías de la Información y de la Comunicación (TIC). Se destacan las gestiones y acciones más sobresalientes de organismos internacionales, al igual que aquellas gestionadas por instituciones panameñas dirigidas hacia la implementación de las TIC con el fin de mejorar la calidad de la educación a nivel superior. Se describen los cambios generados por las nuevas Tecnologías de la Información y de la Comunicación en el sistema educativo a nivel mundial, vaticinando la transformación del proceso de enseñanza-aprendizaje. Finalmente, se detalla el esfuerzo que realiza el Estado Panameño en la introducción de las TIC a nivel superior, con una descripción de la trayectoria de la educación virtual en diferentes universidades a nivel nacional.

\section{Antecedentes Internacionales: Un enfoque mundial}

\subsection{Políticas Internacionales}

El uso de las Tecnologías de la Información y de la Comunicación (TIC) repercute en la modernización del sistema educativo, de manera significativa, y acorta la brecha de aprendizaje en la sociedad del conocimiento. Su evolución es veloz y así se percibe en toda América Latina, el Caribe, América del Norte y Europa.

Al respecto, Guerra, Hilbert, Jordan y Nicolai (2008) han explicado que las aplicaciones de las TIC iniciaron con proyectos de educación a distancia o teleeducación, que luego evolucionaron a la educación electrónica/e-educación (eLearning en inglés), incluyendo aspectos como aprendizaje y enseñanza por medios electrónicos, capacitación para su uso, adquisición de sistemas de aprendizaje y programas educacionales, a través de entornos virtuales de aprendizaje, y el uso de tecnologías de redes y comunicaciones para diseñar, seleccionar, administrar, entregar y extender la educación, así como para organizar y administrar la información relativa a sus educandos.

Según Guerra, Hilbert, Jordan y Nicolai (2008), las racionalidades² económica, social y educativa guían la introducción de las TIC al sistema escolar. La racionalidad económica indica que

\footnotetext{
${ }^{2}$ Racionalidades se refiere a la maximización de beneficio, optimización, según el razonamiento económico, social y educativo
} 
las TIC son necesarias en la educación para que los estudiantes desarrollen las competencias de manejo de las TIC que les serán demandadas en el mundo del trabajo, lo que a su vez permitirá a los países mejorar la competitividad de sus trabajadores, sus empresas y su economía. (Guerra, Hilbert, Jordan y Nicolai, 2008, p.22).

Según la racionalidad social, es imperativo proveer a todos los sectores sociales de un país con las competencias, los recursos y las herramientas necesarias para utilizar las TIC con igualdad de oportunidades para todos. La racionalidad educativa, a su vez, aclara que la introducción de las TIC tiene el propósito de mejorar y transformar las prácticas pedagógicas, dejando atrás las tradicionales clases frontales y moviendo el proceso educativo hacia la pedagogía de índole constructivista, convirtiendo al alumno en un investigador activo y creador del conocimiento (Guerra, Hilbert, Jordan y Nicolai, 2008).

En la misma línea, debido a las relaciones entre el hombre, la sociedad, la vida y el mundo, se deben brindar las condiciones en todos los niveles educativos y, en este caso, a nivel superior, que faciliten la formación y la adquisición de competencias ligadas a los procesos sociales, comunitarios, económicos, políticos, religiosos, deportivos, ambientales y artísticos donde están inmersas las personas, quienes generan experiencias de autorrealización, interacción social y vinculación laboral. Este enfoque, conocido como el enfoque socio formativo complejo, desarrolla la formación humana basada en competencias, el conocimiento complejo o complejidades y expectativas sociales y las potencialidades humanas con respecto a la convivencia y a la producción (Tobón, 2006).

Claro (2010) reafirmó las potencialidades de las TIC en el sector educativo haciendo énfasis en su efectividad para la enseñanza y aprendizaje de diversas asignaturas, y para el desarrollo de las competencias y habilidades de orden superior, tales como: la reflexión, el análisis crítico, el razonamiento, la evaluación que trascienden a las disciplinas tradicionales y que facilitan la resolución de problemas, el aprendizaje cooperativo o colaborativo, y la creación de conocimientos; es decir, la construcción del conocimiento mediado por la tecnología o tecnoconstructivismo. Para Thornburg (2003, citado en García, Hernández, Zúñiga, Charpentier y Carrillo, 2010), la contribución que el individuo realiza en la construcción de su propio conocimiento de manera autónoma es llamado tecno constructivismo, donde la tecnología, o las TIC, se integran dentro del plan de estudios para redefinirlo. 
Para que se generen estas capacidades, se requieren cambios o transformaciones tanto en la gestión como en la modernización de los establecimientos educativos y actualización de los planes de estudios integrando el tecnoconstructivismo (García, Hernández, Zúñiga, Charpentier y Carrillo, 2010). En primer lugar, según Claro (2010), uno de estos cambios está directamente relacionado con la eficiencia de la gestión educativa, ya que se ven involucrados procesos de recolección, monitoreo y análisis de datos sobre establecimientos escolares e instituciones a nivel superior, por el intercambio de información entre la administración central y la administración local, actividades de organización, desarrollo profesional y comunicación de docentes y directivos, procesos de organización e implementación del currículum, estrategias de enseñanza y aprendizaje y la comunicación entre profesor y estudiante, entre otros.

En segundo lugar, Fullan (2001) ha establecido que otro de los cambios se refiere a la búsqueda de innovaciones en los sistemas educativos para que estos pasen de ser centros cerrados y rígidos a centros abiertos y flexibles, donde la colaboración y la transferencia de conocimientos genere nuevas experiencias de aprendizaje, nuevas formas de trabajo, nuevas maneras de interacción para la apropiación y enriquecimiento de los nuevos conocimientos en todos los niveles: primario, secundario y a nivel superior. Estas nuevas formas de construcción de conocimientos deben cumplir con aspectos que generen actitudes y destrezas activas, constructivas, colaborativas, acción intencionada, un intercambio permanente de ideas y de conocimientos en forma contextualizada con proyectos que reflejen la realidad cotidiana y que aborden la reflexión (Díaz, 2006, citado en García, Hernández, Zúñiga, Charpentier y Carrillo, 2010).

Estas nuevas prácticas de las TIC en Educación se apoyan en los temas sobre la obtención de nuevos aprendizajes (OECD, 2001; Proyecto World Links citado en Claro 2010; Banco Mundial, citado en Claro 2010; Harrison et.al., 2003 (citado en Claro, 2010); Osborne y Hennesy, 2003; OECD, 2004; Trucano, 2005; Condie y Munro, 2007, Cabral y Severín, 2010; UNESCO, 2008; OEI, 2010); en el cambio e innovación pedagógica (Quesada, 2010; Becker, 2000; OECD, 2001; Proyecto World Links citado en Claro 2010, Banco Mundial, citado en Claro 2010, Kozma, 2008; Law et. al., 2008, UNESCO, 2008), en las aplicaciones de nuevos recursos como las herramientas de las web 2.0 que permiten crear, diseñar, elaborar, colaborar y publicar en línea (OEI, 2010; Giugni y Araujo, 2010), y en el cambio o innovación organizacional (OECD, 2001; Proyecto World Links citado en Claro 2010; Banco 
Mundial, citado en Claro 2010; Trucano, 2005; Condie y Munro, 2007; Severin (BID, 2010); UNESCO, 2008; OEI. 2010). Los principales modelos de definición e identificación de estas buenas prácticas se apoyan en la diversificación, la promoción, el estudio, la evaluación y el desarrollo de políticas y programas por cuatro organismos internacionales: la Organización de las Naciones Unidas para la Educación, la Ciencia y la Cultura (UNESCO), el Banco Mundial, la Organización para la Cooperación y el Desarrollo Económico (OCDE) y el Banco Interamericano de Desarrollo (BID).

Para la UNESCO, las prácticas de las TIC dependen de su integración exitosa en las salas de clases con la implementación de estructuras de ambientes de aprendizaje notradicionales, de la unión de nuevas tecnologías con nuevas pedagogías en ambientes virtuales de aprendizaje, del desarrollo de clases socialmente activas, del fomento de la interacción cooperadora, el trabajo cooperativo y el trabajo grupal. De igual manera, la UNESCO considera que uno de los factores de mayor impacto se fundamenta en los estándares de competencias TIC para el profesor, desde el enfoque de alfabetización digital o tecnológica y profundización del conocimiento hasta llegar a la creación del conocimiento. Similarmente, Claro (2010) se refiere a estos estándares como

consistentes con los objetivos de desarrollo del milenio definidos por las Naciones Unidas y especifican los cambios que implica para cada componente del sistema educativo: política, currículum y evaluación, pedagogía, uso de la tecnología, organización y administración escolar, y desarrollo profesional docente. (p.10)

El Banco Mundial, a través de su programa World Links, hace hincapié en el desarrollo de competencias en las TIC tanto en estudiantes como en docentes o profesores. Sin embargo, la finalidad del Banco Mundial se define principalmente en "la capacitación del profesor para crear, incorporar y facilitar la innovación en las prácticas de la sala de clases que integren la tecnología de redes, el trabajo en equipo y la Internet en el currículum" (Claro, p. 11). Muy similar a la UNESCO, el Banco Mundial busca innovar en seis ejes: (1) Ministerios de Educación para el desarrollo de un plan estratégico de TIC, (2) desarrollo profesional del profesor, (3) impacto en el estudiante mediante las metodologías para el uso de TIC en las que se capacita a los profesores, (4) medidas de generación de recursos para la sustentabilidad de las tecnologías en los establecimientos educativos, (5) implementación de iniciativas de monitoreo y evaluación, (6) desarrollo de capacidad local para construir 
organizaciones locales que ayuden al Ministerio a expandir, mantener y monitorear los programas TIC, permitiendo la sustentabilidad regional y nacional.

Dentro de los intereses del Banco Mundial, existe otro programa llamado Información para el Desarrollo, que considera la implementación de las TIC como una clave central para el desarrollo de los países. Wagner (2005, citado en Sunkel y Trucco, 2010) propuso un marco conceptual que incluye dos grandes áreas: el desarrollo económico y social nacional y el contexto educacional. En el contexto educativo, la intervención de las TIC se focalizan en el currículo, la pedagogía, la evaluación, la infraestructura y la capacitación de los profesores y la educación digital, con resultados muy significativos en los estudiantes (las habilidades y actitudes de las TIC, habilidades de información y de comunicación) y en los profesores (habilidades en el uso las TIC y habilidades pedagógicas relacionadas con la organización, planeamiento y evaluación en los entornos virtuales académicos).

La Organización de Cooperación y el Desarrollo Económico (OCDE) ha planteado un modelo que centraliza al profesor como punto medular de contacto con respecto a las TIC en la educación a distancia, pero el rol principal se le asigna al estudiante, que caracteriza la experiencia del aprendizaje a través de las TIC como el aprendiz digital dentro y fuera del sistema educativo gracias a los recursos y herramientas de comunicación de las TIC, es decir, a través de las plataformas virtuales. Para que el estudiante ejerza este rol, la integración de las TIC le permite el desarrollo del pensamiento creativo e independiente, la resolución de problemas y la administración y evaluación de su propio aprendizaje, convirtiéndolo en un aprendiz autónomo. Al igual que los planteamientos de la UNESCO y el programa del Banco Mundial, es primordial el uso de competencias de orden mayor y la aplicación de estrategias para el manejo de información y la capacidad de realizar juicios y decisiones sobre relevancia y confiabilidad en la información que se accesa en la Internet; es decir, el manejo de la información y su capacidad de "aprender a aprender" (OCDE, 2001).

Por su lado, el Banco Interamericano del Desarrollo (BID) ha elaborado un modelo para la implementación de las TIC que sintetiza los insumos requeridos para las áreas de infraestructura, contenidos, recursos humanos, gestión y políticas de desarrollo. Este marco conceptual intenta apoyar el diseño, la implementación, el monitoreo y la evaluación de proyectos que buscan incorporar las Tecnologías de la Información y de la Comunicación para el logro de mejoras educativas. Lo interesante de este modelo es que muestra las 
etapas de maduración de la implementación de las TIC: emergencia, aplicación, integración y transformación.

Este marco conceptual, sugerido por el BID, considera los siguientes elementos: a) los aprendizajes de los estudiantes como objetivo final de cada intervención; b) las salidas en los resultados esperados en términos de modificación de las prácticas de enseñanzaaprendizaje y el impacto en términos de resultados de aprendizaje de asignaturas y del desarrollo de habilidades de nivel superior y competencias para el siglo XXI; c) las etapas de desarrollo que presenta la incorporación de entornos virtuales de aprendizaje mediados por las TIC en los procesos y sistemas educativos; d) los insumos, entendidos como líneas de acción en infraestructura, contenidos, recursos humanos, gestión, y políticas; e) los procesos o productos, que corresponden a los elementos que se verán modificados por el proyecto y en los cuales debieran evidenciarse las consecuencias de la intervención propuesta y f) el proceso de seguimiento y evaluación del proyecto, incluyendo las fuentes de datos e información adecuadas a cada contexto (Claro, 2010).

Con respecto al punto "c", referente al marco conceptual propuesto por el Banco Interamericano de Desarrollo (BID) que hace hincapié en la incorporación de entornos virtuales de aprendizaje mediados por las TIC en los procesos y sistemas educativos, esta modalidad es la columna vertebral de un aprendizaje virtual a nivel superior. Pero, ¿qué se entiende por un entorno virtual de aprendizaje? El entorno virtual de aprendizaje (EVA), o la comunidad virtual de aprendizaje (CVA) y según Brenson-Lazan (2001), es

un concepto pedagógico que vincula las tecnologías de la información y comunicación con los espacios educativos actuales. Lo anterior supone cambios en los modelos educativos, en los usuarios involucrados en la formación y en los escenarios donde ocurre el aprendizaje. Estos cambios requieren desarrollarse a partir de la construcción de escenarios pedagógicos que revelen la visión de procesos de enseñanza aprendizaje interactivos, lúdicos, comunicativos, y significativos para las personas involucradas. (p. 22, citado en García, Hernández, Zúñiga, Charpentier y Carrillo, 2010)

Por tal razón, según CENT (2004),

deben añadirse planes de desarrollo profesional del profesorado en estrategias didácticas y tecnologías de la información, medidas de apoyo a la innovación educativa y a la generalización de buenas prácticas, estímulos a la producción y distribución de 
materiales formativos de calidad, planes para promover el aumento de la calidad y la cantidad de la comunicación entre profesores y estudiantes en la función tutorial, entre otros. (p. 6)

Indudablemente, el uso de innovaciones pedagógicas, como los entornos virtuales de aprendizaje mediados por las TIC, ha generado que la brecha geográfica sea cada vez más corta y que el acceso a la educación sea cada vez menos un impedimento para aprender (Quesada, 2009; Quesada 2010). Es por ello que la educación a distancia evoluciona a pasos agigantados y no se puede negar que se obtiene aprendizaje de calidad equivalente y, algunas veces, superior al que se da con la educación convencional.

Es evidente que los entornos de aprendizaje virtuales, mediados por las TIC, constituyen una forma totalmente nueva de Tecnología Educativa y ofrecen una compleja serie de oportunidades y tareas a las instituciones de enseñanza de todo el mundo, definiendo el entorno virtual de aprendizaje como un programa informático interactivo de carácter pedagógico que posee una capacidad de comunicación integrada, es decir, que esté asociada a las Nuevas Tecnologías. Por lo tanto, los futuros docentes y los docentes en ejercicio deben formarse y experimentar dentro de entornos educativos el uso innovador de la tecnología (UNESCO, 1998; UNESCO, 2004).

Dando énfasis a la evolución de comunidades o entornos virtuales de aprendizaje, la UNESCO (2002) en un informe acerca de las Tendencias Actuales de la Educación a Distancia hace una síntesis del desarrollo de esta modalidad a distancia y su implementación a nivel mundial por medio de las TIC, que se detallan a continuación.

\subsection{Influencia de las TIC en nuestros continentes}

En el continente africano, las tecnologías interactivas han tenido un valor limitado por la influencia socioeconómica y política de la región, aunque ha habido un incremento en la implementación de la educación a distancia en los últimos cinco años.

En los Estados Árabes, la educación abierta y a distancia es reciente y está menos extendida que en otras regiones a nivel mundial. A nivel superior, la demanda de tipo de educación va en incremento, pero la viabilidad de acceso a una educación a distancia eficiente y eficaz requiere de recursos para satisfacer esta demanda. En Asia y el Pacífico, Australia es el país con mayor desarrollo en el área de las TIC para la educación abierta y a distancia. Los países asiáticos China y Japón han crecido significativamente en programas e 
instituciones de aprendizaje abierto y a distancia, aunque enfrentan barreras en el uso de las TIC por los altos costos de instalación y mantenimiento, más la falta de experiencia y de recursos humanos. Europa es un continente donde la educación a distancia está bastante arraigada En algunos países se ha incorporado la educación dual o b-learning, y se han abierto universidades y campus virtuales compartidos con varias instituciones igualmente virtuales.

En América Latina y el Caribe existe una creciente campaña diseñada para lograr una educación masiva a través de las TIC, que se logró en primera instancia, años atrás, por medio de la televisión educativa. En los últimos años, las modalidades de educación a distancia se han ido acercando más y con mayor compromiso a la comunicación por medio de la computadora (CMC), o por medio de entornos virtuales de aprendizaje con una amplia gama de cursos y programas virtuales que faciliten la educación continua, principalmente a nivel superior.

Con respecto a América del Norte, la educación a distancia se encuentra firmemente enraizada en los sistemas educativos tanto en Canadá como en los Estados Unidos. El apoyo de las TIC ha evolucionado en todos los niveles de educación y ha alcanzado grupos poblacionales alejados, con oportunidades iguales para todos en todos los campos: capacitación, formación profesional, entrenamiento militar, Educación Superior y continua, entre otros. Las modalidades que se utilizan con más frecuencia son las de capacitación a través de la web, comunicación por medio de la computadora, video teleconferencia, transmisión satelital, cursos por televisión y video, entornos virtuales de aprendizaje, entre otros (UNESCO, 2002).

Un proyecto de gran cobertura para la gestión educativa apoyado por las TIC es el Proyecto@lis-Integra, un proyecto clave para el desarrollo de capacidades en Argentina, Chile y Uruguay. El Proyecto @lis-Integra (CEDEL, 2011), aprobado por la Comisión Europea en el marco de la convocatoria @LIS, solidifica políticas públicas para la inclusión de las TIC en el sector educativo. Este proyecto fue ejecutado del 2003 al 2006 y visualizó el desarrollo y fortalecimiento del conocimiento a nivel latinoamericano, por medio del uso apropiado de las TIC en la enseñanza y la administración. Una de sus metas logradas fue la creación de una Red de Centros de Innovación (Cedel) con el fin de promover las TIC como un recurso para la educación continua de docentes y técnicos y la prestación de servicios a instituciones educativas. Este proyecto es aprobado por la Comisión Europea en el marco de 
la convocatoria @LIS (Alianza para la Sociedad de Información-Europa-América Latina),y países como España, Irlanda, Italia, Uruguay, Argentina, y Chile forman parten del mismo, con más de 20 proyectos encaminados a la utilización de entornos virtuales de aprendizaje mediados por las nuevas tecnologías de información y comunicación en actividades educativas y administrativas. Uno de los proyectos más actualizados de Cedel se ha encaminado hacia personas con discapacidad, cuya finalidad es incorporarlas hacia el uso de las TIC gracias a la instauración de un centro de desarrollo de software y herramientas tecnológicas para el aprendizaje significativo (Proyecto @lis-Integra, 2009).

Tomando como base la Declaración Mundial sobre la Educación Superior en el Siglo XXI (UNESCO, 1998), que señala la necesidad de una formación multicultural basada en la calidad, la pertinencia y la cooperación internacional solidaria a través de redes de universidades, la Fundación Universitaria Iberoamericana (FUNIBER) se pronuncia ante un objetivo primordial de esta declaración: "Educación permanente para toda la Vida". La Fundación Universitaria Iberoamericana (FUNIBER) es una fundación que se enmarca dentro del mundo universitario y su formación. Inició en 1997 en España, y en la actualidad cuenta con una red 25 de universidades y más de 45 instituciones a nivel superior. El concepto de FUNIBER consiste en posibilitar la educación no presencial (a distancia) con el único fin de formar personas como iguales con una educación internacional de primer nivel y categoría sin dejar de lado lo que cada país aporta en su individualidad, singularidad y ventajas comparativas. Uno de los ejes de interés de FUNIBER es promover la creación de programas interuniversitarios, donde usuarios accedan a grados académicos de doble titulación, programas de postgrado, diplomados, especializaciones, cursos de extensión, por medio de entornos virtuales de aprendizaje.

A través de los entornos o ambientes virtuales de aprendizaje apoyados por las TIC y las redes interuniversitarias, el programa de FUNIBER impulsa uno de los fundamentos de la Educación Superior "aprender a aprender", con un modelo educativo que facilite el contacto estudiante-profesor, profesor-profesor, estudiante-estudiante, que fomente el aprendizaje colaborativo, que enfoque la educación centrada en el estudiante, que potencie la comunicación interactiva en plataformas virtuales, que estimule el aprendizaje autónomo y responsable (todo individuo puede educarse en el lugar donde vive y trabaja, sea este cual sea), y que personalice el aprendizaje. FUNIBER tiene sedes en España, Italia, Portugal, Argentina, Bolivia, Brasil, Chile, Colombia, Costa Rica, Ecuador, El Salvador, Guatemala, 
Honduras, México, Nicaragua, Perú, Paraguay, Panamá, Puerto Rico, República Dominicana, Uruguay, Venezuela, USA, Angola, Cabo Verde, Mozambique, entre otros.

Igualmente, en el continente Americano existe la Organización Universitaria Interamericana (OUI), cuya fundación se efectuó en 1980. La meta de la OUI es responder a la necesidad de extender las relaciones entre los países de Centro y Sur América, mejorar la información y favorecer la cooperación académica entre las Instituciones de Educación Superior en las Américas, estimulando la comprensión y ayuda mutua, contribuyendo al desarrollo sostenible de los pueblos de las Américas y respetando la libre discusión de ideas.

En la actualidad, la OUI agrupa más de 300 instituciones y asociaciones nacionales universitarias que acogen a más de 5 millones de estudiantes, 500,000 profesores y 600,000 miembros de personal de apoyo. Sus miembros, que constituyen una red exclusiva de asociados, se encuentran en 26 países divididos en nueve regiones: América Central, Brasil, Canadá, el Caribe, el Cono Sur (Argentina, Chile, Uruguay, Paraguay), los Estados Unidos, México y los Países Andinos (Bolivia, Colombia, Ecuador y Perú) (OUI, 2012).

La OUI promueve marcadamente estudios, cursos, tendencias en investigación sobre temas tales como los siguientes: Perspectivas sobre encuentros presenciales y virtuales de doctorados dirigidos a instituciones de Educación Superior y asociaciones universitarias, Promoción y Utilización de la Educación a Distancia (EaD)/TIC, Congreso Iberoamericano sobre Calidad de la Formación Virtual, Construcción y Montajes de sitos web, Desarrollo de Diplomados de Competencias TIC, Conformación de "Redes de Expertos" por línea estratégica, Formación del Civismo Digital, Creación de grupo de expertos y sitio web para foro de Espíritu y Valores, Curso sobre Medios y Tecnologías para la EaD, Encuentro de Disciplinas de Educación a Distancia, Encuentro Internacional Anual Virtual EDUCA, Talleres para la Formación de Facilitadores en cursos en línea, y el Congreso Iberoamericano de Educación Virtual, entre otros (OUI, 2010, Plan de Actividades).

Siguiendo esta línea, la UNESCO (1999), en su informe mundial de la educación, define a los entornos virtuales de aprendizaje como programas pedagógicos interactivos que facilitan la comunicación integrada por estar asociados directamente con las TIC, y señala que los entornos virtuales de aprendizaje constituyen una forma totalmente nueva de Tecnología Educativa que ofrece una compleja serie de oportunidades y tareas a las instituciones de enseñanza de todo el mundo. 
Es claro que los entornos virtuales de aprendizaje aprovechan todas las funcionalidades de las TIC por medio de las herramientas de la web 2.0, con el fin de ofrecer nuevos entornos para la enseñanza y el aprendizaje libre de las restricciones de tiempo, lugar y espacio, cerciorando una continua comunicación virtual acorde con el ritmo de la transformación educativa mundial.

\section{Antecedentes Nacionales: Panamá}

\subsection{Políticas Nacionales: La Trayectoria de las TIC en Panamá}

A nivel de la República de Panamá, el artículo 8 de la Ley 13 del 15 de abril de 1997 (Gaceta Oficial Digital, 2009; Gaceta Oficial, 2005), establece los Lineamientos e Instrumentos para el Desarrollo de la Ciencia, la Tecnología y la Innovación, por medio de la creación de la Secretaria Nacional de Ciencia, Tecnología e Innovación (SENACYT).

El decreto de la Asamblea Legislativa, publicado en la Gaceta Oficial el 18 de abril de 1997, fomenta una estrategia que contribuya equitativamente a la construcción del conocimiento, propiciando la generación, publicación e intercambio de conocimientos a través de la TIC. Por su lado, la resolución de Gabinete N. 104 del 21 de diciembre 2005, establece y adopta el Plan Estratégico Nacional para el Desarrollo de la Ciencia, Tecnología e Innovación 2006-2010. Dentro de sus considerandos, esta resolución manifiesta dos aspectos importantes: primero, que es responsabilidad del Estado Panameño crear condiciones para que la ciencia, la tecnología y la innovación contribuyan a mejorar la calidad de vida del panameño y, segundo, que es necesario estimular la innovación tecnológica como efecto esencial para fortalecer la capacidad del país que exige la economía mundial. Dentro del Plan Estratégico se revela que es poca la incorporación de la tecnología del proceso de enseñanza-aprendizaje y en la organización y divulgación de la información de las universidades.

A pesar de esto, en Panamá se han hecho varios avances con el fin de posibilitar el aprendizaje a nivel superior por medio de las TIC. Según el Informe Nacional sobre Educación Superior (Castillo, 2003), las primeras universidades en implementar educación virtual fueron la Universidad Interoamericana de Educación a Distancia (UNIEDPA), con sede en Panamá, y la Universidad Abierta y a Distancia de Panamá (UNADP).

Por su parte, en 1999, la Universidad Columbus (Columbus University) inició cursos "off campus", cuyo propósito fue brindarles oportunidades a estudiantes universitarios que no 
podían asistir a sus clases regulares por razones laborales u otras, y completar su educación a través de la Internet. Algunas carreras que Columbus University ofrece off-campus son las licenciaturas en Administración Gerencial, Contabilidad y Auditoría, Turismo y Administración Hotelera, Periodismo, Psicología, entre otros. Maestrías en Administración de Empresas con diversas especializaciones, Maestría en Derecho, Maestría en Educación, Master of Arts, etc. (Castillo, 2003).

Bajo la misma línea, otras universidades privadas que comenzaron a ofrecer cursos virtuales son Nova Southeastern University, en 1998, y el Instituto de Estudios Superiores de Monterrey (ITESM, sede en Panamá), en 1999, estos últimos totalmente virtuales. La mayoría de las carreras que ofrece el ITESM son a nivel de maestría y cuenta con 80,000 estudiantes inscritos en cursos virtuales en México y en América Latina. Dentro de este contexto, la Universidad Tecnológica de Panamá (UTP) inauguró su universidad virtual en el año 2000, con la apertura de algunos cursos virtuales dentro de las carreras, cuya finalidad es cumplir con las necesidades de estudiantes que residen en el interior del país. Otras universidades que han incursionado en la virtualización a nivel superior son Florida State University, la Universidad Especializada de las Américas (UDELAS), Universidad Latina de Panamá, la Universidad Latinoamericana de Ciencia y Tecnología (ULACIT) al igual que el ISAE con cursos semipresenciales centrados en el autoaprendizaje. Con respecto a UDELAS, a partir de junio 2003, y con la aprobación del Centro de Educación Virtual, se ofrecieron programas de Postgrado, Maestría y Doctorado (Informe Nacional sobre Educación Superior, Castillo, 2003).

Por su parte, la Universidad de Panamá es promotora de la educación virtual y lo aclara en el Reglamento General de Estudios de Postgrado, donde define la diferencia entre educación presencial, semipresencial y virtual en Capitulo 1, Artículo 1, letra c que dice los estudios a nivel de postgrado pueden brindarse en modalidades educación presencial, semipresencial, a distancia, virtual u otras que establezca el Consejo de Investigación. Este artículo del reglamento explica en el inciso c. 4 que la educación virtual es aquella donde se aplican las nuevas tecnologías a los procesos sincrónicos y asincrónicos de comunicación y enseñanza. Este artículo también incorpora el parágrafo 1 en el que la Universidad de Panamá promoverá la incorporación de las Nuevas Tecnologías de la Información y de la Comunicación (NTICS) a los procesos de enseñanza y aprendizaje a nivel de postgrado (Castillo, 2003). 
En concordancia, existen varios programas que viabilizan la educación virtual en diferentes universidades del país. Uno de estos programas se está desarrollando entre la Universidad de Panamá (Vicerrectoría de Investigación y Postgrado), Facultad de Ciencias de la Educación y Virtual Educa, para la capacitación de docentes que desean desempeñarse en la formación a través de Entornos Virtuales de Aprendizaje. Virtual Educa es una iniciativa de cooperación multilateral en materia de educación, formación e innovación adscrito a la Cumbre Iberoamericana de Jefes de Estado y de Gobierno (OEI), del cual Panamá forma parte. El programa en mención ha tomado como constructo teórico declaraciones de la UNESCO, cuyo marco de referencia enfatiza que

con el advenimiento de las nuevas tecnologías, el énfasis de la profesión docente está cambiando desde un enfoque centrado en el profesor y basado en clases magistrales, hacia una formación centrada principalmente en el alumno dentro de un entorno interactivo de aprendizaje. El diseño e implementación de programas de capacitación docente que utilicen las TIC efectivamente es un elemento clave para lograr reformas educativas profundas y de amplio alcance (UNESCO, 2004).

El objetivo de este programa en la Universidad de Panamá y Virtual Educa es capacitar a profesionales de diferentes áreas disciplinares, en el marco de las competencias de la metodología e-learning, para el ejercicio de la docencia en entornos virtuales. Este programa de especialización, o maestría en entornos virtuales de aprendizaje, encamina a profesionales hacia los nuevos cambios e innovaciones del mundo globalizado de hoy en día, con el fin de responder a las aceleradas demandas de la sociedad del conocimiento, tales como: educación accesible para todos, uso intensivo y extensivo de las tecnologías, especialización y flexibilización del currículo, y apertura a nuevas formas de "aprender a aprender" a nivel superior. Virtual Educa es un ámbito multilteral auspiciado por las siguientes organizaciones: Secretaria General Iberoamericana (SEGIB), Organización de Estados Americanos (OEA), Parlamento Latinoamericano (PARLATINO), UNESCO, Organización Estados Iberoamericanos para la Educación, la Ciencia y la Cultura (OEI), Red Global de Aprendizaje para el Desarrollo (GDLN), Instituto Latinoamericano Comunicación Educativa (ILCE), y Organización Iberoamericana de Juventud (OIJ).

Siguiendo la misma línea, la Universidad Tecnológica de Panamá con el Departamento de Desarrollo Humano, Educación y Cultura de la OEA (Organización de 
Estados Americanos) también se une a la generación de las nuevas eras del conocimiento con la implementación de un Diplomado en Creación de Ambientes Virtuales de Aprendizaje. Dentro de los objetivos de este diplomado está la promoción de ambientes virtuales de aprendizaje para la descentralización, democratización, pertinencia y continuidad de los procesos educativos, la generación de conocimientos y experiencias a través de estos entornos, el diseño de herramientas metodológicas y prácticas pedagógicas en los procesos de educación formal y no formal, y el desarrollo de una cultura tecnológica que sustente el aprendizaje significativo y los procesos de aprender a aprender.

Para otros antecedentes relacionados con el uso de entornos virtuales de aprendizaje mediado por las TIC en Panamá, se toman en cuenta con proyectos desarrollados por el gobierno. Uno de estos proyectos se llama "Conéctate al Conocimiento". Tarté (2006) explica que este proyecto fue una iniciativa del Gobierno de Panamá como una estrategia nacional basada en el aprendizaje significativo y mapas conceptuales para el sistema público de educación básica.

Para ello, se establece una red nacional de colaboración en la construcción del conocimiento. Según Tarté (2006), "en sus primeros dos años de operación, el proyecto ya ha atendido, cerca de 250 escuelas con procesos de formación para sus docentes ofreciendo 140 talleres en los que participaron 3,200 docentes, directores y supervisores. Un beneficio que se extiende aproximadamente a 90,000 niños y sus familias" (p.1). Para lograr resultados óptimos, se creó en cada escuela pública participante una "Aula de Innovación”, dotada de recursos tecnológicos para apoyar todo el proceso. Uno de los objetivos de este programa es utilizar intensa y masivamente las tecnologías de la información y la comunicación al servicio de los aprendizajes.

El Programa "Conéctate al Conocimiento" se enmarcó dentro del Plan Estratégico 2005-2009 del Ministerio de Educación (MEDUCA) de la República de Panamá, con otro programa llamado "Proyecto para la Mejora y Modernización del Sistema de Formación Institucional Permanente de Docentes". El objetivo general de este plan estratégico fue cambiar el sistema educativo vigente por un modelo de calidad, moderno, participativo, eficiente y más equitativo. El plan de acción utilizado fue por una intervención basada en el uso masivo de las Tecnologías de la Información y de la Comunicación (TIC) y la identificación y aprovechamiento de recursos ya existentes, tanto en línea (on line) como en software (off line), cuyo objetivo específico fue mejorar y modernizar el sistema de formación 
institucional permanente de docentes impulsando la descentralización y agilizando la administración educativa. Los beneficiarios fueron 200 docentes y administrativos en la modalidad presencial, y 600 en la modalidad en línea o a distancia, lo cual demuestra la cobertura de formación educativa a través de las TIC.

Otro proyecto del Ministerio de Educación es "Educa Panamá". El Ministerio de Educación de Panamá ha diseñado un portal que forma parte de la Red Latinoamericana de Portales Educativos (RELPE), conformada por 18 países. Esta red ha sido concebida como un sistema de almacenamiento y distribución de contenidos educativos en constante expansión y renovación que servirá de aporte sustancial a favor de la disminución de la brecha digital que afecta a los países de la región y, por ende, el desarrollo académico y cultural de las naciones. Educa Panamá se caracteriza por la cooperación, el intercambio cultural y la contribución para la mejora de la calidad y equidad de la educación. Su principal compromiso es establecer esquemas de trabajos para la interacción académica, social, cultural y recreativa.

Además, el 3 de agosto de 2009, la República de Panamá se integró en la Red lberoamericana de TIC y Educación (RIATE), luego de participar en la V Jornada Iberoamericana de Cooperación Educativa sobre Tecnologías de la Información y la Comunicación, realizada en Santa Cruz-Bolivia del 20 al 24 de abril del mismo año. A raíz de estas reuniones, se estableció que el objetivo principal de RIATE es el desarrollo y fortalecimiento de los sistemas de educación mediante la efectiva incorporación de las TIC, como medio para lograr la equidad educativa y social, la adquisición y aplicación de las competencias digitales propias de los ciudadanos y ciudadanas del siglo XXI y, con ello, mejorar la calidad de vida y los procesos de transformación social que requieren nuestros pueblos. Esta red la conforman países de Centroamérica y Suramérica (Red Iberoamericana de TIC y Educación, 2009).

Lo interesante de la red RIATE es que contempla tanto la alfabetización digital de docentes, como la formación en herramientas TIC para su utilización didáctica o la utilización como vehículo para soportar la formación en áreas no tecnológicas, como pueden ser la educación de personas adultas, la educación inclusiva o la coeducación. Para RIATE la formación del profesorado es una prioridad. Una de las metas de esta red es enmarcar proyectos de colaboración internacional en educación para lograr objetivos de integración y desarrollo conjunto. La extensión de estas acciones así como los contenidos que las 
conforman dependen de las instituciones participantes y de las necesidades detectadas en cada país (RED RIATE, 2009).

En síntesis, el uso de las TIC ha sido un gran avance para la educación en Panamá, ya que ha abierto ventanas a la posibilidad de ampliar la gama de recursos, estrategias didácticas y las modalidades de comunicación que se pueden ofrecer para el mejoramiento, optimización y alcance del quehacer educativo. La educación en Panamá debe sufrir cambios significativos que favorezcan un empoderamiento al aprendizaje debido a las exigencias de la globalización mundial. Tales cambios se visualizan a pasos agigantados a través de los antecedentes mencionados.

\section{Conclusión}

En la búsqueda de nuevos entornos pedagógicos, la Educación Superior ha sufrido transformaciones radicales donde se están incorporando, en forma moderada, las Tecnologías de la Información y de la Comunicación (TIC), a través de entornos virtuales de aprendizaje como un recurso para la construcción de conocimientos significativos. Esta exploración ha generado lo que actualmente conocemos como universidades virtuales.

A raíz de estos cambios, la Educación Superior se enfrenta a un nuevo paradigma de enseñanza y aprendizaje, aquel que implica la utilización e innovación de nuevas metodologías mediadas por las TIC, la incorporación de nuevos roles docentes y educandos en forma de comunidades virtuales con mayor responsabilidad, flexibilidad, colaboración y comunicación, y puesta en práctica de una nueva visión para la construcción del conocimiento con un aumento en la autonomía del alumnado.

Sin embargo, a pesar de la evidente introducción de las TIC en las universidades, la disponibilidad de la infraestructura TIC necesaria y las competencias tecnológicas requeridas tanto por los docentes y por los educandos es limitada en las universidades públicas de nuestro país. En definitiva, para que esta integración sea eficaz y provechosa debe conllevar cambios que van más allá de los medios tecnológicos utilizados. Estos cambios son de tipo pedagógico y didáctico, donde se rompen las barreras del tiempo y el espacio para desarrollar las actividades de enseñanza y aprendizaje. El reto se centra en modos de aprendizaje a través de entornos virtuales a nivel superior y que esta nueva modalidad sea catalizadora para que las universidades innoven no solamente en tecnología, sino en las 
concepciones y practicas pedagógicas que produzcan cambios y renovaciones impactantes que modifiquen el modelo enseñanza aprendizaje universitario en su totalidad.

Sin lugar a dudas, las TIC influyen marcadamente en la Educación a Distancia (eLearning). De hecho, las TIC son los recursos más indispensables para la efectividad de los entornos virtuales de aprendizaje. A pesar de ello, para muchos profesionales a nivel superior es controversial poder aseverar que los entornos virtuales de aprendizaje (EVA), mediados por las TIC, mantienen elevados niveles de prácticas y resultados efectivos en la Educación Superior tanto en calidad como en cantidad de tiempo, en acortar distancias y generar experiencias de aprendizaje similares a las clases presenciales al igual que en el papel que desempeña el estudiante y el profesor. Para los docentes a nivel superior, la incorporación de los entornos virtuales de aprendizaje a nivel superior es un desafío para los modelos tradicionales y los docentes producen resistencias y rechazos naturales al uso de entornos virtuales de aprendizaje por las incertidumbres de su eficacia y eficiencia, y prefieren mantener y conservar las prácticas pedagógicas ya existentes a nivel superior.

Es evidente que la educación a través de entornos virtuales de aprendizaje se ha convertido en una alternativa de educación a nivel superior, porque impacta en la cobertura y oferta de los servicios de educación (acceso a la educación en cualquier lugar y a cualquier hora), por el fortalecimiento del sistema educativo en las modalidades presencial, virtual y a distancia a través del acceso a contenidos y recursos en línea, y por la utilización de las Tecnologías de la Información y de la Comunicación en el contexto educativo.

Indudablemente, las universidades públicas y privadas de nuestro país no escapan a este impacto por lo que han vislumbrado el potencial de estos nuevos entornos de aprendizaje, y poco a poco están incorporando estas nuevas modalidades de aprendizaje en sus ofertas académicas con el fin de suplir las necesidades de la educación continua y de romper las variables clásicas que se enmarcan los modelos tradicionales de aprendizaje (espacio o dimensión entre la persona que ensena y la que aprende).

Por tal razón, el primer capítulo de esta investigación a nivel doctoral, resumido en este artículo, se enfoca en las implicaciones de las nuevas prácticas de los entornos virtuales de aprendizaje considerando las características particulares de las TIC, los elementos que la componen y el rol que juega cada uno de los actores en el proceso educativo, según organismos nacionales e internacionales, con respecto a las competencias necesarias para 
la eficiencia y efectividad de la tecno educación y con respecto al aprendizaje significativo mediado por la tecnología o tecnoconstructivismo.

Se espera que los antecedentes de las TIC, a nivel mundial y a nivel de la República de Panamá, sean un marco de referencia para las futuras publicaciones que se realizarán acerca de los avances de la tesis doctoral titulada "Modelo de Aprendizaje en Entornos Virtuales en la Educación Superior", tales publicaciones pretenden enfocar los lineamientos pedagógicos de los entornos virtuales de aprendizaje a nivel superior, los tipos y medios de interacción en entornos virtuales de aprendizaje a nivel superior, la modalidad curricular requerida para este tipo de aprendizaje, las competencias requeridas por los docentes y los estudiantes en la utilización de entornos virtuales de aprendizaje en la Educación Superior, las estrategias didácticas, y la evaluación o valoración de un modelo de aprendizaje virtual para la efectividad del aprendizaje significativo a nivel superior. El aporte de esta investigación se encamina hacia una capacitación de docentes y estudiantes en entornos virtuales de aprendizaje a nivel superior para el mejoramiento e innovación de los procesos didácticos con el fin de formarlos de acuerdo a las exigencias de la educación del siglo XXI y de favorecer su competitividad nacional e internacionalmente.

\section{Referencias}

Banco Interamericano de Desarrollo (BID). (2012). Banco Interamericano del Desarrollo: TICs

en

educación.

Recuperado

de

http://www.iadb.org/es/temas/educacion/tecnologias-de-la-informacion-y-lacomunicacion-tic-en-la-educacion-en-america-latina-y-el-caribe,6980.html

Brenson-Lazan, Gilbert. (2001). Etapas de desarrollo y facilitación en una comunidad virtual de aprendizaje. AMAUTA International, LLC. Recuperado de: http://www.amauta-international.com/DesarrolloComunidadVirtual.pdf

Cabral, Marcelo y Severín, Eugenio. (2010). TIC en educación: Una innovación disruptiva. Revista BID Educación, 2, 1-8. Recuperado de: http://idbdocs.iadb.org/wsdocs/getdocument.aspx?docnum=35130690

Castillo, Noemi. (2003). Informe nacional sobre Educación Superior. Recuperado de: http://unesdoc.unesco.org/images/0015/001506/150673so.pdf

Centro de estudios para el desarrollo local (CEDEL). (2011). Centro de estudios para el desarrollo local. Recuperado de: http://www.cedelargentina.org/ 
CENT. (2004). Centre d'Educació i noves tecnologies. Selección de un entorno virtual de enseñanza aprendizaje de código fuente abierto para la Universitat Jaume I. Recuperado de: http://cent.uji.es/doc/eveauji es.pdf

Claro, Magdalena. (2010). La incorporación de tecnologías digitales en educación. Modelos de identificación de buenas prácticas. Naciones Unidas. Recuperado de: http://www.eclac.org/publicaciones/xml/8/40278/tics-educacion-buenas-practicas.pdf.

Condle, Rae y Munro, Bob. (2007). The impact of ICT in schools: A landscape review. Recuperado de: http://webarchive.nationalarchives.gov.uk/20101102103654/publications.becta.org.uk//d isplay.cfm?resID=28221

Ferreiro, Ramón y DeNapoli, Anthony. (2006). Un concepto clave para aplicar exitosamente las tecnologías de la educación: Los nuevos ambientes de aprendizaje. Revista Interamericana de Pedagogía: Saberes y Quehaceres del Pedagogo, 8, 121-154.

Fullan, Michael. (2001). The new meaning of educational change. New York: Teachers College Press.

FUNIBER. (2012). Formación a distancia y presencial en proyectos de un ámbito internacional. Recuperado de: http://www.funiber.org/

García, Jacqueline, Hernández, Lidia, Zúñiga, Claudia, Arnáez, Elizabeth, Charpentier, Claudia y Carrillo, María de los Ángeles. (2010). Comunidad virtual de aprendizaje: Un espacio para la formación ambiental. San José, Costa Rica: INIE

Giugni Ch., Dyuna y Araujo M., Belkys. (2010). TIC y educación: Entornos virtuales de calidad en el proceso de enseñanza-aprendizaje. Recuperado de: http://www.chubut.edu.ar/descargas/secundaria/congreso/TICEDUCACION/RLE2312

Araujo.pdf

Guerra, Massiel, Hilbert, Martin, Jordan, Valeria. y Nicolai, Christian. (2008). Panorama digital 2007 de América Latina y el Caribe: Avances y desafíos de las políticas para el desarrollo con las Tecnologías de Información y Comunicaciones. Naciones Unidas. Recuperado de: http://www.eclac.org/publicaciones/xml/6/34726/W210.pdf

Kozma, Robert. (2008). Comparative analysis of policies for ICT in education. International handbook on information technology in education, Recuperado de: http://robertkozma.com/images/kozma comparative ict policies chapter.pdf

Law, Nancy. (2008). ICT, pedagogical innovations and teacher learning: International handbook of information technology in primary and secondary education. New York: Springer.

Marqués, Pere. (2000). Las TIC y sus aportaciones a la sociedad. Recuperado de: http://peremarques.pangea.org/tic.htm 
OECD. (2001). Learning to change-ICT in schools: Paris. Recuperado de: http://tecnologiaedu.us.es/bibliovir/pdf/red8.pdf

OECD. (2004). Tecnologías de la información y de la comunicación: Perspectivas de la OCDE sobre la tecnología de la información: Edición 2004. Recuperado de: http://www.oecd.org/dataoecd/33/4/33986768.pdf

Organización de Estados Iberoamericanos para la Educación, la Ciencia y la Cultura. (2010). 2021: Metas Educativas. La educación que queremos para la generación de los bicentenarios. Recuperado de: http://www.oei.es/metas2021.pdf

Organización Universitaria Iberoamericana. (2012). Iberoamerican observatory of health and citizenship. Recuperado de: http://www.iohc-pt.org/index.php/pt/noticias/74organizacion-universitaria-interamericana-oui

Organización Universitaria Iberoamericana. (2010). Plan de Actividades: OUI 2010. Recuperado de: http://www.ouiiohe.org/es/images/stories/documentos/plan de actividades2010.pdf

Osborne, Jonathan y Hennesy, Sara. (2003). Literature review in science education and the Role of ICT: Promise, problems and future directions. Recuperado de: http://hal.archives-ouvertes.fr/docs/00/19/04/41/PDF/osborne-j-2003-r6.pdf

Panamá. (2009). Gaceta Oficial Digital: SENACYT. Recuperado de: http://www.oas.org/juridico/spanish/mesicic3 pan res229.pdf

Panamá. (2005). Gaceta Oficial: Ley que modifica la ley 13 de 1997, que establece los lineamientos e instrumentos para el desarrollo de la ciencia, la tecnología y la innovación, crea la secretaria nacional de ciencia, tecnología e innovación como institución autónoma y dicta otra. Recuperado de: http://docs.panama.justia.com/federales/leyes/50-de-2005-dec-23-2005.pdf

Panamá. (2012). Educa Panamá: Ministerio de Educación (MEDUCA). Recuperado de: http://www.educapanama.edu.pa/.

Panamá. (2005). Plan estratégico 2005-2009; Ministerio de Educación (MEDUCA). Recuperado de: http://www.meduca.gob.pa/files/general/plan estrategico.pdf

Proyecto @lis-Integra. (2009). Serie de publicaciones del proyecto @lis-INTEGRA. Recuperado de http://www.oei.es/noticias/spip.php?article1467

Quesada, Allen. (2010). Aprendizaje Colaborativo e Interuniversitario en Línea: Una experiencia asíncrona y síncrona. Revista de Lenguas Modernas, 1(12), 197-210.

Quesada, Allen. (2009). Issues for effective distance learning: A challenge in online education. Revista de Lenguas Modernas, 11, 345-362. 
Red RIATE. (2012). Red Iberoamericana de TIC y Educación. Recuperado de: http://cete.dgtve.sep.gob.mx/snovo/index.php?option=com contentyview=articleyid=7:1 -red-iberoamericana-de-tic-y-educacion-riateycatid=22:0eiyltemid=34

Red RIATE. (2009). Red Iberoamericana de TIC y Educación. Recuperado de: http://www.riate.org/index.php?option=com k2\&view=item\&id=8:panam\%C3\%A1-seintegra-a-la-red-iberoamericana-de-tic-y-educaci\%C3\%B3n-riate\&ltemid=57\&lang=es

Severín, Eugenio. (2010). Tecnologías de la Información y la Comunicación (TIC) en Educación: Marco conceptual e indicadores. Recuperado de: http://idbdocs.iadb.org/wsdocs/getdocument.aspx?docnum=35128349

Solari, Adriana y Monge, Germán. (2004). Un desafío hacia el futuro: Educación a distancia, nuevas tecnologías y docencia universitaria. Recuperado de: http://www.ateneonline.net/datos/96 03 Birri Roberto.pdf

Sunkel, Guillermo y Trucco, Daniela. (2010). Nuevas tecnologías de la información y comunicación para la educación en América Latina: Riesgos y oportunidades. CEPAL: División de Desarrollo Social.

Tarte, Gaspar. (2006). Conéctate al conocimiento: Una estrategia de Panamá basado en mapas conceptuales. Recuperado de: http://cmc.ihmc.us/cmc2006Papers/cmc2006p248.pdf

Tobón, Sergio. (2006). Formación basada en competencias: Pensamiento complejo, diseño curricular y didáctica. Colombia: Ecoe Ediciones.

Trucano, Michael. (2005). Knowledge maps: ICT in Education. Information of development Program. New York: The World Bank. Recuperado de: http://www.infodev.org/en/Publication.8.html

UNESCO. (1998). Conferencia mundial sobre la Educación Superior: La Educación Superior en el siglo XXI. Recuperado de: http://sigc.uaroo.mx/Documentos\%20Externos/Educacion\%20Siglo\%20XXI\%20UNESC $\underline{\text { O.pdf }}$

UNESCO. (1999). Normas UNESCO sobre competencias en TIC para docentes. Recuperado de: http://www.oei.es/tic/normas-tic-marco-politicas.pdf

UNESCO. (2002). Aprendizaje abierto y a distancia: Consideraciones sobre tendencias, políticas y estrategias. División de Educación Superior. Recuperado de: http://online.upaep.mx/Aplicaciones/Noticias/noticiasp.php?id=1209594760

UNESCO. (2005). Construir el siglo XXI entre todos. Recuperado de: http://unesdoc.unesco.org/images/0010/001015/101513s.pdf 
UNESCO. (2004). Las tecnologías de la información y la comunicación en la formación docente: Guía de planificación. Recuperado de: http://unesdoc.unesco.org/images/0012/001295/129533s.pdf

UNESCO. (2008). Proyectos de resolución 2008-2009. Recuperado de: http://unesdoc.unesco.org/images/0015/001501/150144s.pdf

Universidad de Panamá. (2012). Virtual Educa. Recuperado de: http://www.up.ac.pa/PortalUp/VicerrectorialnvestigacionPostgrado.aspx?menu=312

Universidad ORT, Uruguay. (2010). Proyecto @lis-Integra. Recuperado de: http://www.ort.edu.uy/index.php?cookie setted=trueyid=AAAIAB

Universidad Tecnológica de Panamá. (2012). Recuperado de: http://facultad.pucp.edu.pe/comunicaciones/images/documentos/DIPLOMADOCREACI ONAMBIENTESVIRTUALES.pdf

Virtual Educa. (2012). Virtual Educa: Innovación, competitividad, desarrollo. Recuperado de: http://virtualeduca.org/quienes somos.htm

Wagner, Daniel. (2005). Monitoring and evaluation of ICT in education projects. A handbook for developing countries. Washington DC: InfoDev/World Bank. 